\title{
Learning Geometric Concepts with Nasty Noise
}

\author{
Ilias Diakonikolas \\ University of Southern California \\ USA \\ diakonik@usc.edu
}

\author{
Daniel M. Kane \\ University of California, San Diego \\ USA \\ dakane@cs.ucsd.edu
}

\author{
Alistair Stewart \\ University of Southern California \\ USA \\ stewart.al@gmail.com
}

\begin{abstract}
We study the efficient learnability of geometric concept classes - specifically, low-degree polynomial threshold functions (PTFs) and intersections of halfspaces - when a fraction of the training data is adversarially corrupted. We give the first polynomial-time PAC learning algorithms for these concept classes with dimensionindependent error guarantees in the presence of nasty noise under the Gaussian distribution. In the nasty noise model, an omniscient adversary can arbitrarily corrupt a small fraction of both the unlabeled data points and their labels. This model generalizes wellstudied noise models, including the malicious noise model and the agnostic (adversarial label noise) model. Prior to our work, the only concept class for which efficient malicious learning algorithms were known was the class of origin-centered halfspaces.

At the core of our results is an efficient algorithm to approximate the low-degree Chow-parameters of any bounded function in the presence of nasty noise. Our robust approximation algorithm for the Chow parameters provides near-optimal error guarantees for a range of distribution families satisfying mild concentration bounds and moment conditions. At the technical level, this algorithm employs an iterative "spectral" technique for outlier detection and removal inspired by recent work in robust unsupervised learning, which makes essential use of low-degree multivariate polynomials.

Our robust learning algorithm for low-degree PTFs provides dimension-independent error guarantees for a class of tame distributions, including Gaussians and, more generally, any logconcave distribution with (approximately) known low-degree moments. For LTFs under the Gaussian distribution, using a refinement of the localization technique, we give a polynomial-time algorithm that achieves a near-optimal error of $O(\epsilon)$, where $\epsilon$ is the noise rate. Our robust learning algorithm for intersections of halfspaces proceeds by projecting down to an appropriate low-dimensional subspace. Its correctness makes essential use of a novel robust inverse independence lemma that is of independent interest.
\end{abstract}

\section{CCS CONCEPTS}

- Theory of computation $\rightarrow$ Machine learning theory;

Permission to make digital or hard copies of all or part of this work for personal or classroom use is granted without fee provided that copies are not made or distributed for profit or commercial advantage and that copies bear this notice and the full citation on the first page. Copyrights for components of this work owned by others than ACM must be honored. Abstracting with credit is permitted. To copy otherwise, or republish, to post on servers or to redistribute to lists, requires prior specific permission and/or a fee. Request permissions from permissions@acm.org.

STOC'18, fune 25-29, 2018, Los Angeles, CA, USA

(C) 2018 Association for Computing Machinery.

ACM ISBN 978-1-4503-5559-9/18/06 . .\$15.00

https://doi.org/10.1145/3188745.3188754

\section{KEYWORDS}

PAC Learning, Polynomial Threshold Functions, Intersections of Halfspaces, Malicious Noise, Nasty Noise

\section{ACM Reference Format:}

Ilias Diakonikolas, Daniel M. Kane, and Alistair Stewart. 2018. Learning Geometric Concepts with Nasty Noise. In Proceedings of 50th Annual ACM SIGACT Symposium on the Theory of Computing (STOC'18). ACM, New York, NY, USA, 13 pages. https://doi.org/10.1145/3188745.3188754

\section{INTRODUCTION}

\subsection{Background and Motivation}

One of the central challenges in machine learning is to make accurate inferences from datasets in which pieces of information are corrupted by noise. In this work, we study the efficient learnability of geometric concepts - specifically, low-degree polynomial threshold functions and intersections of linear threshold functions (halfspaces) - when a fraction of the training data is adversarially corrupted. As our main contribution, we give the first polynomial-time PAC learning algorithms for these concept classes with dimensionindependent error guarantees ${ }^{1}$, when a constant fraction of the data is arbitrarily corrupted.

Polynomial Threshold Functions (PTFs) and intersections of Linear Threshold Functions (LTFs) are two fundamental classes of Boolean functions that have been extensively studied in many contexts for at least the past five decades $[10,34,35]$. In the noiseless setting, low-degree PTFs are known to be efficiently PAC learnable under arbitrary distributions via linear programming [33]. The current state-of-the-art for PAC learning intersections of LTFs is as follows: Even without noise, distribution-independent PAC learning for intersections of 2 LTFs is one of the most challenging open problems in computational learning theory. Efficient algorithms are known for PAC learning intersections of any constant number of LTFs under well-behaved distributions, e.g., under the standard Gaussian distribution $[39,40]$. Dealing with (adversarial) noisy data turns out to be significantly more challenging in general. Recent results (see, e.g., $[7,8]$ ) provide strong evidence that learning with adversarial noise is computationally intractable under arbitrary distributions, even for simple concept classes.

In this paper, we focus on the efficient learnability of low-degree PTFs and intersections of (any constant number of) LTFs in the presence of nasty noise. In the nasty noise model [4], an omniscient adversary can arbitrarily corrupt a small constant fraction of both the unlabeled data points and their labels. Formally, we have the following definition:

\footnotetext{
${ }^{1}$ By the term "dimension-independent" error guarantee it is meant that: when the fraction of corruptions is $\epsilon$, our algorithms achieve error $f(\epsilon)$ (for some function $f: \mathbb{R}_{+} \rightarrow \mathbb{R}_{+}$such that $\left.\lim _{x \rightarrow 0} f(x)=0\right)$.
} 
Definition 1.1 (Nasty Noise Learning Model). Let $C$ be a class of Boolean-valued functions over $\mathbb{R}^{n}, D$ a distribution over $\mathbb{R}^{n}$, and $f$ an unknown target concept $f \in C$. For $0<\epsilon<1 / 2$, we say that a set $T$ of $m$ labeled examples is an $\epsilon$-corrupted set of examples from $C$ if it is obtained using the following procedure: First, we draw a set $S=\left\{\left(x^{(i)}, y_{i}\right)\right\}$ of $m$ labeled examples, $1 \leq i \leq m$, where for each $i$ we have that $x^{(i)} \sim D, y_{i}=f\left(x^{(i)}\right)$, and the $x^{(i)}$ 's are independent. Then an omniscient adversary, upon inspecting the set $S$, is allowed to remove an $\epsilon$-fraction of the examples and replace these examples by the same number of arbitrary examples of its choice. The modified set of labeled examples is the $\epsilon$-corrupted set $T$. A learning algorithm in the nasty noise model is given as input an $\epsilon$-corrupted set of examples from $C$ and its goal is to output a hypothesis $h$ such that with high probability the error $\operatorname{Pr}_{x \sim D}[h(x) \neq f(x)]$ is small.

The nasty model generalizes a number of well-studied noise models, including the malicious noise model [26, 37] and the agnostic (adversarial label noise) model [21, 27]. Specifically, in the malicious model, the adversary can add an $\epsilon$-fraction of corrupted labeled examples, but is not allowed to adversarially remove good labeled examples. ${ }^{2}$ In the adversarial label noise model, the adversary can corrupt an $\epsilon$-fraction of the labels of $f$ under $D$, but cannot change the distribution $D$ of the unlabeled points. We formally define these models and elaborate on the relation between them in Section 1.3. While these noise models were originally defined with respect to arbitrary distributions, it has been recently shown [7] (modulo plausible complexity assumptions) that, even for the class of LTFs, no computationally efficient algorithm can achieve dimension-independent error guarantees. Hence, research in this area has focused on noise-tolerant learning under a number of "tame" distributions.

Our goal in this paper is to design polynomial-time robust learning algorithms that can tolerate nasty noise of constant rate, i.e., we want to achieve error guarantees that are independent of the dimension.

We now briefly review prior work in this area. In the agnostic (adversarial label noise) PAC model [21, 27], the $L_{1}$-regression algorithm of Kalai et al. [22] can be used to learn low-degree PTFs and intersections of LTFs under a number of well-behaved distributions, including the uniform distribution on the hypercube and the standard Gaussian distribution; see, e.g., [12, 19, 20, 23-25, 29]. The algorithmic technique of [22] achieves the information-theoretically optimal noise tolerance, but it leads to algorithms with runtime $n^{\text {poly }(1 / \epsilon)}-$ where $n$ is the dimension and $\epsilon$ the error rate - even for a single LTF under the Gaussian distribution. A sequence of subsequent works $[1,6,28]$ focused on designing $\operatorname{poly}(n, 1 / \epsilon)$ time learning algorithms that can tolerate constant noise rate in the malicious model (and the adversarial label noise model) with respect to well-behaved continuous distributions. The culmination of this line of work [1] was that the concept class of origin-centered LTFs (i.e., halfspaces whose separating hyperplane goes through the origin) is efficiently learnable in the malicious model - with error guarantee of $O(\epsilon)$ for noise rate $\epsilon$ - under isotropic (i.e., zero-mean, identity covariance) log-concave distributions.

\footnotetext{
${ }^{2}$ This distinction is important. Recent work $[15,17]$ has shown computational separations between these models in a related unsupervised learning setting.
}

Perhaps surprisingly, the concept class of origin-centered LTFs is the only family of Boolean functions for which polynomial-time algorithms are known in the malicious model. This motivates the following broad question that was posed as an open problem in previous work $[1,28]$ :

\begin{abstract}
Are there computationally efficient learning algorithms in the malicious noise model - with dimension-independent error guarantees - for more general classes of Boolean functions?
\end{abstract}

\subsection{Overview of Our Contributions}

In this paper, we study this question with a focus on more general geometric concept classes, namely low-degree PTFs and intersections of a constant number of LTFs. We provide new algorithmic and analytic techniques that yield the first polynomial-time PAC learning algorithms for these concept classes in the nasty noise model (hence, in the malicious model as well) with dimension-independent error guarantees.

Specifically, we give a robust learning algorithm for low-degree PTFs in the nasty model that succeeds under a number of wellbehaved distributions - including the Gaussian distribution and, more generally, any log-concave distribution with (approximately) known low-degree moments. Prior to our work, no non-trivial efficient learning algorithm was known (even) for degree-2 PTFs in the (weaker) malicious noise model. As an additional implication of our techniques, we also obtain the first efficient nasty learning learning algorithm with dimension-independent error for LTFs under the uniform distribution on the hypercube.

For LTFs under the Gaussian distribution, using additional ideas, we give a polynomial-time algorithm that achieves error $O(\epsilon)$, where $\epsilon$ is the noise rate, i.e., it matches the information-theoretically optimal error, up to a constant factor. This is the first malicious/nasty learning algorithm for the class of arbitrary LTFs that achieves error $O(\epsilon)$ in polynomial time. Our result improves on prior work by Awasthi et al. [1] in two respects: First, [1] achieved an $O(\epsilon)$ error bound for the special case of origin-centered LTFs, and second their algorithm applies to the weaker malicious/agnostic models. On the other hand, the $O(\epsilon)$ bound of [1] holds for the more general family of isotropic log-concave distributions.

Our third main result is a polynomial-time learning algorithm with dimension-independent error guarantees for intersections of (any constant number of) LTFs in the nasty model under the Gaussian distribution. To the best of our knowledge, no efficient algorithm (with non-trivial error guarantees) was previously known even for intersections of 2 LTFs in the (weaker) malicious noise model.

At the core of the aforementioned results is an efficient algorithm to approximate the low-degree Chow parameters of any bounded function in the presence of nasty noise. Roughly speaking, the lowdegree Chow parameters of a function $f$ under a distribution $D$ are the "correlations" of $f$ (with respect to $D$ ) with all low-degree monomials (see Section 1.5 for the formal definition). Our algorithm succeeds for a range of reasonable distributions $D$ satisfying mild concentration bounds and moment assumptions. At a high-level, our robust (low-degree Chow parameter estimation) algorithm employs an iterative spectral technique for outlier detection and 
removal, inspired by recent work in robust unsupervised learning [13]. Our technique filters out corrupted points relying on the concentration of carefully chosen low-degree polynomials.

Our robust learning algorithms for PTFs and intersections of LTFs use our Chow-parameters estimation algorithm as a basic subroutine. That is, for both concept classes, our algorithms proceed in two steps: (1) We start by approximating the "low-degree" Chow parameters of our function, and (2) We use our approximate Chow parameters from Step (1) to find a proper hypothesis that is close to the target concept.

The algorithm for Step (2) differs for PTFs and intersections of LTFs. For degree- $d$ PTFs, we use the fact that approximations to the degree- $d$ Chow parameters information-theoretically approximately determines our function. Given this fact, we leverage known algorithmic techniques $[9,36]$ that allow us to efficiently find an accurate proper hypothesis with approximately these Chow parameters. For intersections of $k$ LTFs, we rely on approximations to the degree-2 Chow parameters. In this case, these parameters allow us to reduce our $n$-dimensional learning problem to a $(k+1)$-dimensional problem that we can efficiently solve by a simple net-based method. The correctness of this scheme crucially relies on a novel structural result about intersections of LTFs under the Gaussian distribution that we believe is of broader interest.

\subsection{Noise Models}

We start by recalling the standard (noiseless) PAC learning model [38]: Let $C$ be a class of Boolean-valued functions over $\mathbb{R}^{n}$. We assume that there exists a fixed distribution $D$ over $\mathbb{R}^{n}$ and an unknown target concept $f \in C$. The learning algorithm is given a set $S=\left\{\left(x^{(i)}, y_{i}\right)\right\}$ of $m$ labeled examples $\left(x^{(i)}, y_{i}\right), 1 \leq i \leq m$, where for each $i$ we have that $x^{(i)} \sim D, y_{i}=f\left(x^{(i)}\right)$, and the $x^{(i)}$ 's are independent. The goal of the algorithm is to output a hypothesis $h$ such that with high probability the error $\operatorname{Pr}_{x \sim D}[h(x) \neq f(x)]$ is small.

In this work, we consider the problem of learning geometric concepts in the nasty (noise) model [4], which we now describe. As in the PAC model, a distribution $D$ over $\mathbb{R}^{n}$ is selected and a target concept $f \in C$ is chosen. The critical difference with the noiseless PAC model lies in how the labeled examples given to the learning algorithm are constructed. In the nasty model, the examples that the algorithm gets are generated by a powerful adversary that works according to the following steps: First, the adversary chooses $m$ examples independently according to the distribution $D$. Then the adversary, upon seeing the specific $m$ examples that were chosen (and using his knowledge of the target function, the distribution $D$, and the learning algorithm), is allowed to remove a fraction of the examples and replace these examples by the same number of arbitrary examples of its choice. The points not chosen by the adversary remain unchanged and are labeled by their correct labels according to $f$. The modified labeled sample $S^{\prime}$ of size $m$ is then given as input to the learning algorithm. The only restriction is that the adversary is allowed to modify at most an $\epsilon$-fraction of examples. As in the PAC model, the goal of the algorithm is to output a hypothesis $h$ such that with high probability the error $\operatorname{Pr}_{x \sim D}[h(x) \neq f(x)]$ is as small as possible. The information-theoretically optimal error achievable in the nasty model is well-known to be $\Theta(\epsilon)$ [4].
We will be interested in designing efficient learning algorithms in the nasty model, i.e., algorithms with sample complexity and running time $\operatorname{poly}(n, 1 / \epsilon)$ that achieve error $f(\epsilon)$, where $f: \mathbb{R}_{+} \rightarrow \mathbb{R}_{+}$ is a function such that $\lim _{x \rightarrow 0+} f(x)=0$. In other words, we want the error guarantee of our learning algorithm to be independent of the dimension. The "golden standard" in this setting is to achieve $f(\epsilon)=O(\epsilon)$, i.e., to match the information-theoretic limit, up to a constant factor.

As is well-known, the nasty noise model generalizes both the malicious noise model [26, 37] and the agnostic (adversarial label) model [21, 27]. In the malicious model, each labeled example is generated independently as follows: With probability $1-\epsilon$, a random pair $(x, y)$ is generated where $x \sim D$ and $y=f(x)$; and with probability $\epsilon$ the adversary can output an arbitrary point $(x, y) \in \mathbb{R}^{n} \times\{-1,1\}$. Each of the adversary's examples can depend on the state of the learning algorithm and the previous draws of the adversary. Hence, in the malicious model, the adversary can add corrupted labeled samples but cannot remove good labeled examples. In the adversarial label noise model, the adversary can corrupt an $\epsilon$-fraction of the labels of $f$ under $D$, but cannot change the distribution $D$ of the unlabeled points.

\subsection{Related and Previous Work}

We now summarize the prior work that is most relevant to the results of this paper.

As mentioned in the preceding discussion, the malicious noise model with respect to arbitrary distributions is known to be very challenging computationally. Even for the class of $n$-dimensional LTFs, the only known efficient algorithm [26] achieves an error of $\Omega(\epsilon n)$, where $\epsilon$ is the noise rate. Improving on this bound has remained a challenge for a long time, and it was recently shown [7] that this holds for a reason: under plausible complexity assumptions, no efficient algorithm can achieve error at most $1 / 2-1 / n^{c}$, for some constant $c>0$, even if $\epsilon$ is an arbitrarily small constant.

Due to the computational difficulty of malicious learning under arbitrary distributions, research on this front has focused on well-behaved distributions. The prior results most relevant to this paper are the works of Klivans et al. [28] and Awasthi et al. [1]. Klivans et al. [28] studied the problem of learning origin-centered LTFs in the malicious and adversarial label noise models, when the distribution on the unlabeled samples is uniform over the unit sphere or, more generally, an isotropic log-concave distribution. [28] gave the first polynomial-time algorithms for these problems with error guarantee poly-logarithmic in the dimension. For the uniform distribution, their algorithm achieves error $O(\sqrt{\epsilon} \log (n / \epsilon))$, where $\epsilon$ is the malicious noise rate. Under isotropic log-concave distributions, they achieve error $O\left(\epsilon^{1 / 3} \log ^{2}(n / \epsilon)\right)$. These bounds were subsequently significantly improved by [1] who gave a poly $(n, 1 / \epsilon)$ time algorithm that learns to accuracy $O(\epsilon)$, where $\epsilon$ is the malicious/adversarial label noise rate, for isotropic log-concave distributions. Origin-centered LTFs are the only concept class for which efficient malicious learning algorithms were previously known.

At the technical level, the algorithm of [28] uses a simple outlier removal method to approximate the degree-1 Chow parameters, and then finds an LTF with approximately these Chow parameters. (That is, the high-level approach of our work for learning degree- $d$ PTFs 
is a broad generalization of the [28] approach.) It is worth noting that the outlier removal procedure of [28] is a weaker version of the filtering technique from [13]. On the other hand, the algorithm of [1] uses a soft outlier removal procedure together with localization. Instead of using degree-1 Chow parameters, [1] uses hinge-loss minimization, which can be solved via a convex program.

The problem of learning intersections of 2 LTFs under arbitrary distributions (without noise) is one of the most notorious open problems in computational learning theory: No efficient algorithm is known despite decades of effort and it is a plausible conjecture that the problem may be intractable. In a sequence of works, Vempala $[39,40]$ gave $\operatorname{poly}_{k}(n, 1 / \epsilon)$ time algorithms to PAC learn intersections of $k$ LTFs under log-concave distributions on $\mathbb{R}^{n}$. As we will explain in Section 2, our algorithm for learning intersections of LTFs in the nasty model has some similarities with [39], and can be roughly viewed as a robust version of this algorithm. It is also known $[2,30]$ that intersections of $k=2$ origin-centered LTFs are efficiently PAC learnable under isotropic log-concave distributions. We note that these algorithms work in the noiseless PAC learning model. In the agnostic model, the $L_{1}$-regression algorithm [22] can learn an intersection of $k=O(1)$ LTFs under tame distributions, though its running time is $n^{\text {poly }(1 / \epsilon)}$, even for the case of $k=1$. Prior to our work, we are not aware of any non-trivial algorithms for this concept class in the adversarial label/malicious noise model that run in time $\operatorname{poly}(n, 1 / \epsilon)$, even for $k=2$.

Finally, we remark that our work is related to a sequence of recent results on robust estimation in the unsupervised setting [1316]. Specifically, our general algorithm to approximate the lowdegree Chow parameters with nasty noise is inspired by the outlier removal technique of [13]. We emphasize however that the setting considered here is vastly more general than that of [13]. As a result, a number of new conceptual and technical ideas are required, that we introduce in this paper.

\subsection{Preliminaries}

We record the basic notation and definitions used throughout the paper.

Notation. For $n \in \mathbb{Z}_{+}$, we denote by $[n]$ the set $\{1,2, \ldots, n\}$. For $d \in \mathbb{Z}_{+}$, a degree- $d$ polynomial threshold function (PTF) over $\mathbb{R}^{n}$ is a Boolean-valued function $f: \mathbb{R}^{n} \rightarrow\{ \pm 1\}$ of the form $f(x)=$ $\operatorname{sign}(p(x))$, where $p: \mathbb{R}^{n} \rightarrow \mathbb{R}$ is a degree- $d$ polynomial with real coefficients. For $d=1$, we obtain the concept class of Linear Threshold Functions (LTFs) or halfspaces. An intersection of $k$ halfspaces is any function $f: \mathbb{R}^{n} \rightarrow\{ \pm 1\}$ such that there exist $k$ LTFs $f_{i}, i \in[k]$, with $f(x)=1$ iff $f_{i}(x)=1$, for all $i$. For a degree$d$ polynomial $p: \mathbb{R}^{n} \rightarrow \mathbb{R}$, we denote by $\|p\|_{2}$ its $L_{2}$-norm, i.e., $\|p\|_{2}=\mathbf{E}_{x \sim D}\left[p(x)^{2}\right]^{1 / 2}$, where the intended distribution $D$ over $x \in \mathbb{R}^{n}$ will be clear from the context. We say that $p$ is normalized if $\|p\|_{2}=1$. We say that a set of labeled samples is $\epsilon$-corrupted if it is generated in the nasty model at noise rate $\epsilon$, i.e., the adversary is allowed to corrupt an $\epsilon$-fraction of samples.

We now define the degree-d Chow parameters of a function with respect to a distribution $D$. We will require the following notation: Let $m(x)$ be the function that maps a vector $x \in \mathbb{R}^{n}$ to all the monomials of $x$ of degree at most $d$. Concretely, let $\mathbf{a}^{1}, \ldots, \mathbf{a}^{\ell}$ be an enumeration of all $\mathbf{a} \in \mathbb{N}^{n}$ with $\|\mathbf{a}\|_{1} \leq d$. We set $m_{i}(x) \stackrel{\text { def }}{=}$ $\prod_{j=1}^{n} x_{j}^{\mathbf{a}_{j}^{i}}$, for all $1 \leq i \leq \ell$. Now $m$ is a function from $\mathbb{R}^{n}$ to $\mathbb{R}^{\ell}$ with $\ell \leq(n+1)^{d}$.

Definition 1.2 (Chow Parameters). Let $f: \mathbb{R}^{n} \rightarrow[-1,1]$ be a bounded function over $\mathbb{R}^{n}$ and let $D$ be a distribution over $\mathbb{R}^{n}$. The degree-d Chow parameters of $f$ with respect to $D$ are the $\ell$ numbers $\mathbf{E}_{x \sim D}\left[f(x) m_{i}(x)\right]$, for $1 \leq i \leq \ell$.

We will also need an appropriate notion of approximation for the Chow parameters of a function $f: \mathbb{R}^{n} \rightarrow[-1,1]$ :

Definition 1.3 (Chow Parameter Approximation). We say that a vector $v \in \mathbb{R}^{\ell}$ approximates the degree- $d$ Chow parameters of $f$ within Chow distance $\delta$ if the following holds: For all normalized degree- $d$ polynomials $p: \mathbb{R}^{n} \rightarrow \mathbb{R}$ with $p(x)=\sum_{i=1}^{\ell} a_{i} m_{i}(x)$, we have that $\left|L(p)-\mathbf{E}_{X \sim D}[p(X) f(X)]\right| \leq \delta$, where $L(p)=\sum_{i=1}^{\ell} a_{i} v_{i}$ is the corresponding linear combination of our approximations.

Let $p_{1}, \ldots, p_{\ell}$ be an orthonormal basis for the set of degree- $d$ polynomials under the inner product defined by $D$. We note that the previous definition is equivalent to the $\ell_{2}$-distance between the vectors $\left(L\left(p_{i}\right)\right)_{i=1}^{\ell}$ and $\left(\mathbf{E}_{X \sim D}\left[p_{i}(X) f(X)\right]\right)_{i=1}^{\ell}$ being at most $\delta$.

\subsection{Our Results}

We start by stating our core efficient procedure that approximates the low-degree Chow parameters of any bounded function under tame distributions in the presence of nasty noise:

Theorem 1.4 (Estimation of Low-Degree Chow Parameters With NAsty NoISE). Let $f: \mathbb{R}^{n} \rightarrow[-1,1]$. There is an algorithm which, given $d \in \mathbb{Z}_{+}, \epsilon>0$, and a set $S$ of $\operatorname{poly}\left(n^{d}, 1 / \epsilon\right) \epsilon$-corrupted labeled samples from a distribution $D$ over $\mathbb{R}^{n}$, where $D$ is either (a) the standard Gaussian distribution $N(0, I)$ or the uniform distribution $U_{n}$ over $\{ \pm 1\}^{n}$, or (b) any log-concave distribution over $\mathbb{R}^{n}$ with known moments of degree up to $2 d$, runs in $\operatorname{poly}\left(n^{d}, 1 / \epsilon\right)$ time and with high probability, outputs approximations of $\mathbf{E}_{X \sim D}\left[f(X) m_{i}(X)\right]$ for all degree at most $d$ monomials $m_{i}(x)$, such that for any normalized degree-d polynomial $p: \mathbb{R}^{n} \rightarrow \mathbb{R}$, the approximation of $\mathbf{E}_{X \sim D}[f(X) p(X)]$ given by the corresponding linear combination of these expectations has error at most $\epsilon \cdot O_{d}(\log (1 / \epsilon))^{d / 2}$ in case (a) and at most $\epsilon \cdot O_{d}(\log (1 / \epsilon))^{d}$ in case $(b)$.

Theorem 1.4 applies (with appropriate parameters) to a wide range of distributions over $\mathbb{R}^{n}$, as its proof requires only mild tail bounds and moment assumptions. See Definition 3.1 and Proposition 3.2 for detailed statements.

We believe that the algorithmic problem of robustly approximating the low-degree Chow parameters ("Fourier coefficients") of a Boolean function is interesting in its own right and may be useful in other settings as well. Given that a range of classical supervised learning algorithms rely on Fourier coefficients (see, e.g., $[31,32])$, it is perhaps surprising that this problem has not been more systematically investigated in the literature for $d>1$.

We note that the error guarantee of Theorem 1.4 is informationtheoretically optimal up to logarithmic factors, even for the $d=1$ case. This follows from the observation that an $\epsilon$-approximaton of 
the Chow parametrs for LTFs yields an $O(\epsilon)$-approximation in $L_{1^{-}}{ }^{-}$ distance, and the fact that $\Omega(\epsilon) L_{1}$-error is information-theoretically required for any learning algorithm in the nasty model [4].

We now proceed to state our algorithmic results on nasty PAC learning of geometric functions. Our first result is an efficient learning algorithm for low-degree PTFs in the nasty noise model:

Theorem 1.5 (Learning Low-Degree PTFs with NAsty Noise). There is a polynomial-time algorithm for learning degree-d PTFs in the presence of nasty noise with respect to $N(0, I)$ or any logconcave distribution in $\mathbb{R}^{n}$ with known moments of degree at most $2 d$. Specifically, if $\epsilon$ is the noise rate, the algorithm runs in poly $\left(n^{d}, 1 / \epsilon\right)$ time and outputs a hypothesis degree-d PTF $h(x)$ that with high probability satisfies $\operatorname{Pr}_{X \sim D}[h(X) \neq f(X)] \leq \epsilon^{\Omega(1 / d)}$, where $f$ is the unknown target PTF.

This is the first efficient algorithm for learning degree- $d$ PTFs, for any $d>1$, in the malicious/nasty noise model with dimensionindependent error guarantees. The algorithm of Theorem 1.5 works in two stages: We start by approximating the degree- $d$ Chow parameters of our PTF $f$ (using the algorithm of Theorem 1.4), and then we employ (an adaptation of) known techniques $[9,36]$ to find a PTF $h$ with approximately these Chow parameters. The fact that $\operatorname{Pr}_{X \sim D}[h(X) \neq f(X)]$ will be small follows from the following claim: For the considered distributions, approximation in "Chow distance" implies approximation in $L_{1}$-distance. This claim holds for all distributions $D$ such that $p(D)$ has non-trivial concentration and anti-concentration properties for all degree- $d$ polynomials $p$.

It should be noted that the special case of Theorem 1.5 for $d=1$ (LTFs) is already a generalization of [1], in the sense that our result applies to all LTFs (not necessarily origin-centered). For $d=1$, our algorithm requires knowledge of the first 2 moments of the underlying log-concave distribution, which is equivalent to assuming isotropic position (as is done in [1]). For $d=1$ under isotropic log-concave distributions, the final accuracy of our algorithm will be $O(\sqrt{\epsilon})$, while [1] obtains an $O(\epsilon)$ error bound for origin-centered LTFs. Finally, we remark that for $d=1$, Theorem 1.5 also holds under the uniform distribution on the hypercube, with a quantitatively worse - but still dimension-independent - error of $2^{-\Omega(\sqrt[3]{\log (1 / \epsilon)})}$. This follows by using the structural result of [9] relating closeness in Chow distance and $L_{1}$-distance in the Boolean domain.

For LTFs under the Gaussian distribution, the algorithm of Theorem 1.5 can be shown to give final $L_{1}$-error of $O(\epsilon \sqrt{\log (1 / \epsilon)})$. For this important setting, using additional algorithmic ideas, we obtain an efficient algorithm with near-optimal error guarantee:

Theorem 1.6 (Near-Optimally Learning LTFs with Nasty NoISE). There is a polynomial-time algorithm with near-optimal error tolerance for learning LTFs in the presence of nasty noise with respect to $N(0, I)$. Specifically, if $\epsilon$ is the noise rate, the algorithm runs in $\operatorname{poly}(n, 1 / \epsilon)$ time and outputs a hypothesis LTF $h(x)$ that with high probability satisfies $\operatorname{Pr}_{X \sim D}[h(X) \neq f(X)] \leq O(\epsilon)$, where $f$ is the unknown target LTF.

Our algorithm for Theorem 1.6 starts from the $O(\epsilon \sqrt{\log (1 / \epsilon)})$ approximate LTF of Theorem 1.5 and uses a new twist of the localization technique of [1] to reduce the error down to $O(\epsilon)$. We note that a number of new ideas are required here to make this approach work for all LTFs with near-optimal error - as opposed to only origin-centered ones - and to be able to handle nasty noise.

In addition to resolving the efficient learnability of LTFs in the nasty noise model (under the Gaussian distribution), Theorem 1.6 carries an intriguing conceptual message. In the related setting of robustly learning the mean $\mu$ of an identity covariance Gaussian $N(\mu, I)$, recent work has established a computational separation between the nasty model and the malicious model. Specifically, any computationally efficient Statistical Query (SQ) algorithm ${ }^{3}$ for robust mean estimation must have error $\Omega(\epsilon \sqrt{\log (1 / \epsilon)})$ [17] in the nasty model; while there is an efficient (SQ) algorithm that achieves the optimal $O(\epsilon)$ error in the malicious model [15]. Perhaps surprisingly, we show that such a separation does not appear in our supervised setting, even though the unlabeled data points can be adversarially corrupted. Intuitively, this holds for the following reason: While an adversary can fool a robust mean estimation algorithm by appropriately corrupting the tails of the underlying distribution, a robust LTF learning algorithm can focus its attention near the boundary of the separating hyperplane, hence is not affected by such corruptions.

Our third algorithmic result gives the first efficient learning algorithm for intersections of LTFs in the malicious/nasty noise model:

Theorem 1.7 (LEARNING INTERSECTIONS OF LTFS With NASTY NoISE). There is a polynomial-time algorithm for learning intersections of any constant number of LTFs in the presence of nasty noise with respect to $N(0, I)$. Specifically, if $\epsilon$ is the noise rate, the algorithm uses poly $(n, k, 1 / \epsilon)$ labeled examples, runs in $\operatorname{poly}_{k}(n, 1 / \epsilon)$ time, and outputs a hypothesis intersection of $k$ LTFs $h(x)$ that with high probability satisfies $\operatorname{Pr}_{X \sim D}[h(X) \neq f(X)] \leq \operatorname{poly}(k) \cdot \operatorname{poly}(\epsilon)$, where $f$ is the unknown target concept.

Our algorithm establishing Theorem 1.7 works as follows: After approximating the degree- 2 Chow parameters of $f$, we give a relatively simple method to reduce the problem down to $k$ dimensions. The algorithm is quite simple, but its analysis is not. Specifically, the correctness of our dimension-reduction scheme makes essential use of the following new structural result, that we believe is of broader interest:

Theorem 1.8 (Robust InVERSE Independence For InTERSECTIONS OF LTFs). Let $f: \mathbb{R}^{n} \rightarrow\{0,1\}$ be the indicator function of an intersection of $k$ LTFs. Suppose that there is some unit vector $v$ so that for any degree at most 2 polynomial $p: \mathbb{R} \rightarrow \mathbb{R}$ with $\mathbf{E}_{X \sim N(0,1)}[p(X)]=0$ and $\mathbf{E}_{X \sim N(0,1)}\left[p^{2}(X)\right]=1$, and a standard multivariate Gaussian $G \sim N(0, I)$, we have that $|\mathrm{E}[f(G) p(v \cdot G)]|<$ $\delta$. Then, if $G$ and $G^{\prime}$ are standard multivariate Gaussians that are correlated to be the same in the directions orthogonal to $v$ and independent in the $v$-direction, we have that $\mathrm{E}\left[\left|f(G)-f\left(G^{\prime}\right)\right|\right] \leq$ $\operatorname{poly}(\delta) \cdot \operatorname{poly}(k)$.

Note that the indicator of an intersection of $k$ halfspaces $f$ only depends on $k$ linear functions of the input. Hence, for any $v$ perpendicular to all relevant directions, $f(G)$ is uncorrelated with $p(v \cdot G)$ for any function (and in particular polynomial) $p$. Theorem 1.8 is a robust inverse version of this fact: Let $v$ be a direction such that $f$

${ }^{3}$ We note that all known algorithms for the problems under consideration fit into the SQ learning model. 
is nearly uncorrelated with $p(v \cdot G)$ for all degree-2 polynomials $p$. Then, $f$ is nearly constant in the $v$-direction.

\subsection{Organization}

The structure of this extended abstract is as follows: In Section 2, we provide a detailed outline of our techniques. In Section 3, we give our algorithm to robustly estimate the low-degree Chow parameters of a bounded function, thereby establishing Theorem 1.4. Due to space limitations, the remaining algorithms and proofs can be found in the full version of this paper [18].

\section{DETAILED OVERVIEW OF OUR TECHNIQUES}

In this section, we give a detailed outline of our techniques in tandem with a comparison to previous work.

\subsection{Robust Estimation of Low-Degree Chow Parameters}

All our robust PAC learning results hinge on a new algorithm to approximate the degree- $d$ Chow parameters of any bounded function with respect to a sufficiently nice distribution $D$, even under noise in the nasty model (see Proposition 3.2). Before we explain the ideas underlying this algorithm, we elaborate on the metric in which these approximations are guaranteed to be "close". To motivate our choice of metric, we will first discuss another interpretation of the degree- $d$ Chow parameters of a function $f$. In particular, these parameters encode a linear functional mapping degree at most $d$ polynomials $p$ to the expectation $\mathbf{E}_{X \sim D}[p(X) f(X)]$. It is natural to put a norm on Chow parameters that is the dual of the $L_{2}$ norm on polynomials $p$ (with respect to $D$ ). In particular, when we say that we have approximated the degree- $d$ Chow parameters of $f$ to within error $\delta$, we will mean that we have found a linear functional $L$ mapping degree at most $d$ polynomials to real numbers so that for any normalized degree- $d$ polynomial $p$, we have that $\left|L(p)-\mathbf{E}_{X \sim D}[p(X) f(X)]\right| \leq \delta$.

We start by noting that if we had access to noiseless samples, the desired approximation would be easy to perform. In particular, we could take $L(p)$ to be the empirical expectation of $p(x) f(x)$, and then - so long as $D$ satisfies even mild concentration bounds with sufficiently many samples it is straightforward to show that this will be a good approximation with high probability. It turns out that so long as we have reasonably good tail bounds for $p(D)$, this empirical approximation also works well even against noise in the adversarial label (agnostic) noise model. This holds essentially because changing the value of $f$ on a small number of sample points can only have a large impact on the expectation of $p(x) f(x)$ if $p$ is especially large a decent fraction of the time.

The situation becomes substantially more challenging when the noise can adversarially corrupt the unlabeled examples as well, and in particular in the nasty noise model. The essential problem here is that the error in the values allows an adversary to produce many sample values where $p(x)$ is unusually large for some particular $p$, and this will - almost regardless of the labels of these points cause substantial errors in the empirical expectation of $p(x) f(x)$. In order to circumvent this obstacle, we will need a technique for detecting and removing these outliers, and for this we will make use of a "filter" technique inspired by recent work on robust distribution learning [13].

The basic idea here is that if the algorithm knew which polynomials $p$ the adversary was trying to corrupt, it could simply remove all of the sample points for which $p(x)$ was too large, thus removing these errors. Unfortunately, every sample $x$ will have $p(x)$ be abnormally large for some polynomials $p$, so our algorithm will need to find a way to identify particular polynomials for which our expectation may have been substantially corrupted. In order to achieve this, we note that since there must be many erroneous points for which $|p(x)|$ is large, this will cause the empirical expectation of $p^{2}(x)$ to be substantially larger than it should be. This anomaly can be detected (assuming that the algorithm knows good approximations to the true $2 d^{t h}$ moments of $D$ ) by a spectral technique, namely an eigenvalue computation. If such a $p$ is found then, assuming good tail bounds on the distribution of $p(D)$, the fact that we have many data points with much larger values of $p(x)$ than should be likely, will allow us to find a large set of samples most of which are corrupted. This step essentially produces a strictly cleaner version of our original corrupted sample set, and by iterating this algorithm we eventually reach a point where there are no longer any bad polynomials. At this point, we can show that the empirical approximation of the Chow parameters will be accurate.

Although the basic intuition outlined above is in line with recent works $[13,15]$ making use of the filter technique, there are a number of crucial technical differences in our setting. The first of these is that we are now working in a much more general context. Previous works tended to make very specific assumptions about the underlying distribution (e.g., Gaussian or balanced product distribution). Here, we are only making assumptions about tail bounds of higher-degree polynomials. Importantly, existing works typically only needed to ensure that the expectations of degree- 1 and 2 polynomials were correct, while in our setting we will inherently need to use a "filtering" (outlier removal) technique dealing with polynomials of larger degrees. We also run into a new technical complication in the initial steps of the algorithm. In order to get the filtering technique to work, we need to begin by throwing away all of the "extreme" outliers. This is required for a number of reasons involving showing that the necessary concentration bounds hold. In previous works, the criteria for identifying these extreme outliers were generally fairly simple (e.g., throwing away a point being too far from the mean in some appropriate metric). However, in our case, we have less structure to deal with, and therefore need a somewhat more general criterion. In particular, we throw away outliers where $|p(x)|$ is too large for any normalized degree- $d$ polynomial $p$.

Our robust algorithm for low-degree Chow parameter estimation has immediate applications for robustly learning the Chow parameters over a distribution $D$, if $D$ is a Gaussian, Bernoulli, or log-concave distribution (where in the latter case, the algorithm must also know the low-degree moments of $D$ ). In the following paragraphs, we explain how to apply this algorithm as a core subroutine to robustly PAC learn geometric concept classes. 


\subsection{Robust Learning for Low-Degree PTFs}

One of the most natural geometric families of Boolean functions to consider is that of PTFs. By classic structural results $[3,5]$, we know that any degree- $d$ PTF is uniquely determined by its degree- $d$ Chow parameters. This suggests that if we can learn the degree- $d$ Chow parameters of a degree- $d \operatorname{PTF} f(x)$ to sufficient accuracy, then we may be able to use them to learn $f$ itself. In fact, by known algorithmic results $[9,36]$ we know that this is essentially the case but with one slight wrinkle. Since we will only have approximations to the degree- $d$ Chow parameters of $f$, we will need a robust version of the $[3,5]$ structural theorem. That is, we will need to know that if two degree- $d$ PTFs have $L_{1}$-distance at least $\epsilon$, then they must have Chow distance at least $g(\epsilon)$ for some reasonably large error function $g$. While in the case of the uniform distribution over the hypercube establishing such a result is still a challenging open question for $d>1$, it is not hard to prove good bounds when $D$ is a Gaussian, or more generally, a log-concave distribution. For these distributions, we establish that the error function $g$ is proportional to $\epsilon^{d+1}$. This gives an algorithm for properly learning a PTF over one of these distributions to error $\tilde{O}\left(\epsilon^{1 /(d+1)}\right)$, even with $\epsilon$ error in the nasty model.

We note that, for large constant $d$, one cannot expect to do substantially better than this bound using only an approximation of the degree- $d$ Chow parameters. This is because there are pairs of degree- $d$ PTFs for which this $\epsilon$ vs. $\epsilon^{1 / d}$ type relation is nearly tight. This suggests some sort of "integrality gap" getting in the way: No generic algorithm will be able to learn the low-degree Chow parameters of an $\epsilon$-noisy PTF to error better than $\epsilon$, and no generic algorithm will be able to learn a degree- $d$ PTF to error better than $\epsilon^{1 / d}$ from its degree- $d$ Chow parameters. However, this is not the case for the special case of linear threshold functions, where $L_{1}$-distance and Chow distance are indeed proportional.

\subsection{Optimally Robust Learning of LTFs}

For the case of LTFs, the relation between Chow distance and $L_{1}$-distance allows for the possibility of a much better algorithm: that of learning LTFs to an optimal $O(\epsilon)$ error. In fact, we give such an algorithm over the Gaussian distribution. We note that a direct application of the ideas of the previous paragraph is already sufficient to obtain an error of only $O(\epsilon \sqrt{\log (1 / \epsilon)})$. Removing the final logarithmic term requires several new ideas. The overarching principle in our new algorithm is to use the localization technique of [1], though with slightly different technical backing.

Our algorithm will run an initial first pass to obtain an $O(\epsilon \sqrt{\log (1 / \epsilon)})$ approximation to $f$. This step approximates $f$ by an LTF with separator given by some hyperplane $H$. We will then perform rejection sampling on our inputs in order to simulate samples from another Gaussian distribution centered around $H$. Learning $f$ with respect to this new input distribution will allow us to refine our original guess.

There are two major impacts of our restriction procedure. The first is that if most of the erroneous samples are near $H$, they might survive the rejection sampling process with higher probability than other points. This means that the fraction of errors in our simulated sample set may be much larger. To compensate for this though, this restriction will amplify the effect of small errors in $f$, as moving away from $H$ now much more quickly moves one away from the center of the distribution. This means that learning even rough information about the restriction of $f$ will give us useful information about the original problem. These two effects, as it turns out nearly cancel each other out, with the exception that the $\sqrt{\log (1 / \epsilon)}$ term in the error becomes a $\sqrt{\log (1 / \delta)}$, where $\delta$ is the (now much larger) error rate for the restricted distribution. By iterating this technique with thinner and thinner restrictions, we can eventually converge on $f$ to an error of only $O(\epsilon)$.

\subsection{Robust Learning of Intersections of LTFs}

We give a robust algorithm for learning intersections of LTFs with respect to the Gaussian distribution. This algorithm is very different than the one for PTFs, as it is not possible to recover such a function from its low-degree Chow parameters directly. For this problem, we will need to make use of a somewhat different idea. The key insight is that if $f$ is the indicator function of an intersection of $k$ halfspaces, then $f$ only depends on $k$ linear functions of the input. If we could identify these directions, we could project our inputs down to a $k$-dimensional subspace and proceed by applying even relatively inefficient algorithms to learn a function on this lowdimensional space. To learn this subspace, we note that for any $v$ perpendicular to all relevant directions, $f(G)$ is uncorrelated with $p(v \cdot G)$ for any function (and in particular polynomial function) $p$. If we knew the degree- 2 Chow parameters of $f$, this would imply that $v$ was a null-vector of the associated matrix. This would allow us to easily identify such vectors $v$.

In order to turn this into an algorithm, we will first need an inverse version of this theorem. Namely, that if for some vector $v$ that the function $f$ is uncorrelated with $p(v \cdot G)$ for all degree-2 polynomials $p$, we have that $f$ is in fact independent of the $v$-direction. In fact, since we only know approximations to the Chow parameters, we will need a robust version of this statement. Namely if for all degree-2 polynomials $p$, we have that $f$ is nearly uncorrelated to $p(v \cdot G)$, then in fact $f$ will be nearly constant in the $v$-direction.

The proof of this structural result is quite technical, but the basic idea is simple: The projection of $f \cdot G$ onto the line parallel to $v$ is a log-concave (and thus unimodal) function times a Gaussian. If $\mathbf{E}[f(G) p(v \cdot G)]$ is 0 for all such $p$, this means that this projection must be a Gaussian times a constant. However, this puts us in the equality case of the fact that the projection of a log-concave function is log-concave. A careful examination of this condition shows that this is impossible, unless $f$ is constant on lines parallel to $v$. The proof of our structural result is a robust version of this argument.

The aforementioned robust structural result allows a very natural algorithm: We start by learning approximations of the degree- 1 and 2 Chow parameters of $f$. We then let $V$ be the subspace spanned by the vector of degree-1 Chow parameters and the largest $k$ eigenvalues of the matrix corresponding to the degree- 2 Chow parameters. It is not hard to see that $f$ is nearly uncorrelated to $p(v \cdot G)$ for any $v \perp V$. This along with the above structural result allows us to approximate $f(x)$ by a function that depends only on the projection $\pi_{V}(x)$, which as described above, can be learned by brute-force methods. 
We note that the algorithm of [39] for finding the $k$-dimensional invariant subspace is similar to ours. Instead of considering the largest eigenvalues of the degree-2 Chow parameters, the algorithm of [39] relies on the smallest eigenvalues of the covariance of the positive samples, which is roughly equivalent. The correctness of this algorithm uses the following lemma: in the $k$-dimensional subspace in which the intersection is non-trivial, the variance of the positive samples is less than one, which has some similarities with our structural result. The major difference is that our structural lemma is robust, and as a result our algorithm can tolerate nasty noise (using our approximations to the Chow parameters).

\section{ROBUST ESTIMATION OF LOW-DEGREE CHOW PARAMETERS}

In this section, we give our generic algorithm to robustly approximate the degree- $d$ Chow parameters of any bounded function over $\mathbb{R}^{n}$. Our algorithm succeeds for any distribution $D$ over $\mathbb{R}^{n}$ that satisfies mild concentration and moment conditions. We will show that in order to approximate the Chow parameters of degree at most $d$, it suffices to run a filter algorithm that attempts to make the moments of the distribution close to what they should be. To do this, it is enough to have approximations to the moments up to degree $2 d$ and to know tail bounds for polynomials of degree at most $d$.

Specifically, we introduce the following definition:

Definition 3.1 (Reasonable Distribution). We say that a probability distribution $D$ over $\mathbb{R}^{n}$ is reasonable if it satisfies the following conditions:

(i) (Concentration) A tail bound for all degree at most $d$ polynomials: that is, a function $Q_{d}(T)$ such that for all polynomials $p(x)$ with $\|p\|_{2} \leq 1, \operatorname{Pr}_{X \sim D}[|p(X)| \geq T] \leq Q_{d}(T)$.

(ii) (Known Approximations of Low-Degree Moments) A matrix $\Sigma$ such that $(1-\gamma) \mathbf{E}_{X \sim D}\left[m(X) m(X)^{T}\right] \leq \Sigma \leq(1+$ $\gamma) \mathbf{E}_{X \sim D}\left[m(X) m(X)^{T}\right]$, for some relative error $\gamma>0$ that is smaller than a sufficiently small constant.

(iii) A parameter $\delta>0$ that satisfies $\delta \geq \int_{0}^{\infty} T \min \left\{\epsilon, Q_{d}(T)\right\} d T$. Intuitively, the parameter $\delta$ is the maximum amount by which an $\epsilon$-probability mass can contribute to the $\mathbf{E}_{X \sim D}\left[p^{2}(X)\right]$.

(iv) A threshold $T_{\max }$ such that $Q_{d}\left(T_{\max } / 2 \sqrt{\ell}\right) \leq \epsilon /(10 \ell)$ and $T_{\max } \geq \sqrt{\ell}$. We will be able to ignore points $x$ with $|p(x)| \geq$ $T_{\max }$.

We will see in the next section that many common distributions satisfy this definition (for appropriate parameters), including the Gaussian distribution, log-concave distributions, the uniform distribution over the hypercube, etc.

Now we can state the main proposition from which our main algorithmic applications will follow:

Proposition 3.2. Let $D$ be a reasonable distribution with known parameters $Q_{d}(T), \Sigma, \delta$, and $T_{\max }$. There is an algorithm that, given $d \in \mathbb{Z}_{+}, \epsilon>0$, and a set $S^{\prime}$ of $\epsilon$-corrupted labelled samples from $D$ of size $\left|S^{\prime}\right|=\Theta\left(n^{d} T_{\max }^{4} / \epsilon^{2}\right)$, runs in poly $\left(\left|S^{\prime}\right|\right)$ time, and with probability at least $9 / 10$ outputs approximations of $\mathbf{E}_{X \sim D}\left[f(X) m_{i}(X)\right]$ for all monomials $m_{i}(x)$ of degree at most $d$, such that for any degree-d polynomial $p(x)$ the approximation of $\mathbf{E}_{X \sim D}[f(X) p(X)]$ given by the corresponding linear combination of these expectations has error at most $O\left(\operatorname{Var}_{X \sim D}[p(X)] \sqrt{\epsilon(\gamma+\delta+\epsilon)}\right)$.

At a high-level, the algorithm works as follows: First, we preprocess our corrupted set of samples $S^{\prime}$ using a basic pruning step. Specifically, we remove samples $x \in S^{\prime}$ such that there is a polynomial of degree at most $d$ with $\|p\|_{2}=1$ and $|p(x)| \geq T_{\max }$. Our main algorithm is an iterative filtering procedure: Using the largest eigenvalue and eigenvector of an appropriate matrix, we can detect whether there is such a polynomial $p$ whose variance is bigger in $S^{\prime}$ than $D$. If there is, we can use the tail bound $Q_{d}(T)$ to find a filter that throws out points where $|p(x)|$ is too large. If there is no such polynomial, then we show that the empirical Chow parameters suffice, so we output those. Formally, the algorithm is the following:

\section{Algorithm Robust-Chow-Parameters}

(1) Remove all points $x$ from $S^{\prime}$ that have $m(x)^{T} \Sigma^{-1} m(x) \geq T_{\max }^{2} / 2$.

(2) Repeat the following until no more points are removed from $S^{\prime}$ :

(a) Compute the matrix $M$ $\Sigma^{-1 / 2} \mathbf{E}_{X \in \in_{u} S^{\prime}}\left[m(X) m(X)^{T}\right] \Sigma^{-1 / 2}$.

(b) Approximate the largest eigenvalue $\lambda^{*}$ of $M-I$ and the corresponding unit eigenvector $v^{*}$.

(c) If $\lambda^{*} \leq O(\gamma+\delta+\epsilon)$, break, i.e., goto Step 3 .

(d) Consider the polynomial $p^{*}(x)$ $\left(v^{*}\right)^{T} \Sigma^{-1 / 2} m(x)$.

(e) Find $T>0$ such that

$$
\operatorname{Pr}_{X \in \in_{u} S^{\prime}}\left[\left|p^{*}(X)\right| \geq T\right] \geq 4 Q_{d}(T)+3 \epsilon / T_{\max }^{2} .
$$

(f) Remove from $S^{\prime}$ all samples with $\left|p^{*}(x)\right| \geq T$. (3) Return $\mathbf{E}_{X \in_{u} S^{\prime}}\left[f(X) m_{i}(X)\right]$, for all $1 \leq i \leq \ell$.

REMARK 3.3. The algorithm as written assumes that $\Sigma$ is nonsingular. If it is singular, we can find its null vectors. Each of these corresponds to a non-constant polynomial $p(x)$ with $\mathbf{E}_{X \sim D}\left[p(X)^{2}\right]=$ 0 and so with probability $1, p(x)=0$. If we pre-process by removing all points with $p(x) \neq 0$ for all such polynomials, then we can ignore these null-vectors. We can then replace all the inverses in the algorithm with Moore-Penrose pseudo-inverses and still get the same guarantee.

We will now require a definition of a good set, that is a set of points in $\mathbb{R}^{n}$ which satisfies a set of desired properties from a large enough set of random samples from $D$. There is a complication here because our assumptions on $\Sigma$ and $\delta$ only give bounds on moments of degree up to $2 d$. To naively show that $\mathbf{E}_{X \in \in_{u} A}\left[p(X)^{2}\right]$, where $A$ is a set of samples from $D$, is close to $\mathbf{E}_{X \sim D}\left[p(X)^{2}\right]$, we would need bounds on $\operatorname{Var}_{X \in_{u} A}\left[p(X)^{2}\right]$. However, we assume nothing about moments of degree $4 d$. We can get round this by considering the properties of the set after we've thrown away outliers in our pruning step.

Definition 3.4. Let $f: \mathbb{R}^{n} \rightarrow[-1,1]$. We say that a set $S$ of points in $\mathbb{R}^{n}$ is $(\epsilon, f)$-good if for all polynomials $p(x)$ of degree at most $d$ with $\|p\|_{2}=1$ all the following conditions are satisfied: 
(i) For all $T>0$, we have that

$$
\left|\operatorname{Pr}_{X \in \in_{u} S}[p(X)>T]-\operatorname{Pr}_{X \sim D}[p(X)>T]\right| \leq \epsilon /\left(10 T_{\max }^{2}\right) .
$$

(ii) Let $S_{\text {prune }}$ be the subset of points in $S$ that satisfy condition prune, i.e., for all $x \in S_{\text {prune }}$ it holds $m(x)^{T} \Sigma^{-1} m(x) \leq$ $T_{\max }^{2} / 2$. Then, for all $T>0$, we have that

$$
\left|\underset{X \in \in_{u} S_{\text {prune }}}{\operatorname{Pr}}[p(X)>T]-\underset{X \sim D \mid \text { prune }}{\operatorname{Pr}}[p(X)>T]\right| \leq \epsilon /\left(10 T_{\max }^{2}\right) .
$$

(iii) It holds $\left|\mathbf{E}_{X \in_{u} S_{\text {prune }}}[p(X) f(X)]-\mathbf{E}_{X \sim D \mid \text { prune }}[p(X) f(X)]\right| \leq$ $\epsilon$.

A set $S$ that satisfies conditions (i) and (ii) is called $\epsilon$-good.

Before showing that a set of random samples is good, we need a couple of lemmas about our pruning process. Firstly, we show that our pruning indeed implies a bound on the value of the polynomials we consider:

Lemma 3.5. Let $p(x)$ be a degree-d polynomial with $\|p\|_{2} \leq 1$ and $y \in \mathbb{R}^{n}$ be such that $m(y)^{T} \Sigma^{-1} m(y) \leq T_{\max }^{2} /(1+\gamma)$. Then, we have that $|p(y)| \leq T_{\max }$. In particular, this holds for all $y \in \mathbb{R}^{n}$ satisfying $m(y)^{T} \Sigma^{-1} m(y) \leq T_{\max }^{2} / 2$.

Proof. We can write $p(x)=v^{T} \Sigma^{-1 / 2} m(x)$ for some $v \in \mathbb{R}^{\ell}$. Using the bounds on $\Sigma$, we can write

$1 \geq \mathbf{E}_{X \sim D}\left[p(x)^{2}\right]=v^{T} \Sigma^{-1 / 2} \mathbf{E}_{X \sim D}\left[m(X) m(X)^{T}\right] \Sigma^{-1 / 2} v \geq v^{T} v /(1+\gamma)$

Now we have that

$$
\begin{aligned}
p(y) & =v^{T} \Sigma^{-1 / 2} m(y) \leq\|v\|_{2} \cdot\left\|\Sigma^{-1 / 2} m(y)\right\|_{2} \\
& \leq \sqrt{(1+\gamma)} \cdot T_{\max } / \sqrt{(1+\gamma)} \\
& \leq T_{\max },
\end{aligned}
$$

as desired.

We next need to show that the pruning step does not throw away too many points:

Lemma 3.6. We have that: $\operatorname{Pr}_{X \sim D}\left[m(X)^{T} \Sigma^{-1} m(X) \geq T_{\max }^{2} / 2\right] \leq$ $\epsilon / 10$. If $S$ is any set of points satisfying Condition (i) of Definition 3.4, then $\operatorname{Pr}_{X \in u} S\left[m(X)^{T} \Sigma^{-1} m(X) \geq T_{\max }^{2} / 2\right] \leq \epsilon / 5$.

Proof. Let $p_{1}(x), \ldots, p_{\ell}(x)$ be an orthonormal basis for the set of all polynomials of degree at most $d$ under the inner product $\mathbf{E}_{X \sim D}[p(X) q(X)]$ for polynomials $p(x), q(x)$.

From the definition of $T_{\max }$, we have $Q_{d}\left(T_{\max } / 2 \sqrt{\ell}\right) \leq \epsilon /(10 \ell)$. Thus, the probability that for a given $i, 1 \leq i \leq \ell$, it holds $\left|p_{i}(X)\right| \geq$ $T_{\max } /(2 \sqrt{\ell})$ for $X \sim D$ is at most $\epsilon /(10 \ell)$. By our assumption on $S$, the probability of the same event under $S$ is at most $\epsilon /(10 \ell)+$ $\epsilon /\left(10 T_{\max }^{2}\right) \leq \epsilon /(5 \ell)$. By a union bound, the event that there exists $i, 1 \leq i \leq \ell$, such that $\left|p_{i}(X)\right| \geq T_{\max } /(2 \sqrt{\ell})$, for some $1 \leq i \leq \ell$, has probability at most $\epsilon / 10$ under $X \sim D$ and at most $\epsilon / 5$ under $X \in_{u} S$.

Now fix an $x \in \mathbb{R}^{n}$ with $m(x)^{T} \Sigma^{-1} m(x) \geq T_{\max }^{2} / 2$. Consider the polynomial

$$
p(y)=m(x)^{T} \Sigma^{-1} m(y) /\left\|\Sigma^{-1 / 2} m(x)\right\|_{2} .
$$

Then we have that $p(x)=\left\|\Sigma^{-1 / 2} m(x)\right\|_{2} \geq T_{\max } / \sqrt{2}$, and that

$$
\begin{aligned}
& \mathbf{E}_{X \sim D}\left[p(X)^{2}\right]= \\
& m(x)^{T} \Sigma^{-1} \mathbf{E}_{X \sim D}\left[m(X) m(X)^{T}\right] \Sigma^{-1} m(x) /\left\|\Sigma^{-1 / 2} m(x)\right\|_{2}^{2} .
\end{aligned}
$$

Recall that, by our assumption on $\Sigma$, we have

$$
(1+\gamma)^{-1} \Sigma \leq \mathbf{E}_{X \sim D}\left[m(X) m(X)^{T}\right] \leq(1-\gamma)^{-1} \Sigma,
$$

and thus we have

$$
1 /(1+\gamma) \leq \mathbf{E}_{X \sim D}\left[p(X)^{2}\right] \leq 1 /(1-\gamma) .
$$

Thus, we can write $p(x)=\sum_{i=1}^{\ell} a_{i} p_{i}(x)$, where $\|a\|_{2} \leq 1 / \sqrt{(1-\gamma)}$, and therefore $\|a\|_{1} \leq \sqrt{\ell} / \sqrt{(1-\gamma)}$. If all $p_{i}$ 's satisfy

$$
\left|p_{i}(x)\right| \leq T_{\max } /(2 \sqrt{\ell}),
$$

then we would have

$|p(x)| \leq \sqrt{\ell} / \sqrt{(1-\gamma)} \cdot T_{\max } /(2 \sqrt{\ell})<T_{\max } /(2 \sqrt{1-\gamma})<T_{\max } / \sqrt{2}$.

Since $p(x) \geq T_{\max } / \sqrt{2}$, one of these conditions must fail. However, we argued that this event happens with appropriately bounded probabilities under both $S$ and $D$. This completes the proof.

Now we can show that a large enough set of samples drawn from $D$ is $(\epsilon, f)$-good with high probability.

Lemma 3.7. With probability $9 / 10$, if $S$ is a set of $\Omega\left(n^{d} T_{\max }^{4} / \epsilon^{2}\right)$ samples from $D$, then $S$ is $(\epsilon, f)$-good.

Proof. To establish condition (i), we note that the VC-dimension of the set of degree- $d$ PTFs is $O\left(n^{d}\right)$. So, by the VC-inequality [11], with probability $99 / 100$, we have that

$$
\left|\operatorname{Pr}_{X \in u} S[p(X)>T]-\operatorname{Pr}_{X \sim D}[p(X)>T]\right| \leq \epsilon /\left(10 T_{\max }^{2}\right)
$$

for all degree at most $d$ polynomials $p(x)$, and all $T \in \mathbb{R}$. We henceforth condition on this event.

We now proceed to establish that Condition (ii) is satisfied. Lemma 3.6 gives that, for $X \sim D$, we have $m(X)^{T} \Sigma^{-1} m(X) \geq$ $T_{\max }^{2} / 2$ with probability at most $\epsilon / 10$. By our conditioning, the set $S$ satisfies Condition (i). Thus, Lemma 3.6 also implies that for $X \in_{u} S$, we have that $m(X)^{T} \Sigma^{-1} m(X) \geq T_{\max }^{2} / 2$ with probability at most $\epsilon / 5$. Thus, $S_{\text {prune }}$ contains $\Omega\left(n^{d} T_{\max }^{4} / \epsilon^{2}\right)$ samples that can be considered as being drawn from $D$ |prune. Condition (ii) now follows from the same argument as (i) with probability at least 99/100.

For Condition (iii), note that there exists a set of polynomials $p_{1}(x), \ldots, p_{\ell}(x)$ that give an orthonormal basis for the set of all polynomials of degree at most $d$ under the inner product $\mathbf{E}_{X \sim D}[p(X) q(X)]$ for polynomials $p(x), q(x)$. Note that for any $i$, $1 \leq i \leq \ell$, we have

$$
\begin{aligned}
\operatorname{Var}_{X \sim D \mid \text { prune }}\left[p_{i}(X) f(X)\right] & \leq \operatorname{Var}_{X \sim D}\left[p_{i}(X) f(X)\right] /(1-\epsilon / 10) \\
& \leq(1+\epsilon) \mathbf{E}_{X \sim D}\left[p_{i}(X)^{2} f(X)^{2}\right] \\
& \leq 1+\epsilon,
\end{aligned}
$$

where we used the fact that the range of $f$ is $[-1,1]$. Fix $i, 1 \leq i \leq \ell$. By Bernstein's inequality, since we have $\left|S_{\text {prune }}\right|=\Omega\left(T_{\max }^{4} / \epsilon^{2}\right)$, we 
get that

$$
\begin{aligned}
& \operatorname{Pr}\left[\left|\mathbf{E}_{X \in \in_{u} S_{\text {prune }}}\left[p_{i}(X) f(X)\right]-\mathbf{E}_{X \sim D \mid \text { prune }}\left[p_{i}(X) f(X)\right]\right| \geq\left(\epsilon / T_{\text {max }}\right)\right] \\
& \leq \exp \left(-\frac{(1 / 2)\left(\left|S_{\text {prune }}\right| \epsilon / T_{\max }\right)^{2}}{\left|S_{\text {prune }}\right|(1+\epsilon)+(1 / 3) T_{\max } \cdot\left|S_{\text {prune }}\right|\left(\epsilon / T_{\max }\right)}\right) \\
& =\exp \left(-\frac{(1 / 2)\left|S_{\text {prune }}\right| \epsilon^{2}}{T_{\max }^{2}(1+4 \epsilon / 3)}\right) \\
& =\exp \left(-\Omega\left(T_{\max }^{2}\right)\right) \\
& \leq 1 /\left(100 T_{\max }^{2}\right) \\
& \leq 1 /(100 \ell) \text {. }
\end{aligned}
$$

By a union bound, we get that for all $i, 1 \leq i \leq \ell$, it holds

$$
\begin{aligned}
& \left|\mathbf{E}_{X \in_{u} S_{\text {prune }}}\left[p_{i}(X) f(X)\right]-\mathbf{E}_{X \sim D \mid \text { prune }}\left[p_{i}(X) f(X)\right]\right| \\
\leq \quad & \epsilon / T_{\max } \leq \epsilon / \sqrt{\ell},
\end{aligned}
$$

with probability at least $99 / 100$. We condition on this event. Note that any polynomial $p(x)$ with $\|p\|_{2}=1$ can be written as $p(x)=$ $\sum_{i=1}^{\ell} a_{i} p_{i}(x)$, where $\|a\|_{2}=1$, and so $\|a\|_{1} \leq \sqrt{\ell}$. Thus, any such $p(x)$ has

$$
\begin{aligned}
& \left|\mathbf{E}_{X \in_{u} S_{\text {prune }}}\left[p_{i}(X) f(X)\right]-\mathbf{E}_{X \sim D \mid \text { prune }}\left[p_{i}(X) f(X)\right]\right| \\
\leq & \sum_{i=1}^{\ell}\left|a_{i}\right| \epsilon / \sqrt{\ell} \leq \epsilon .
\end{aligned}
$$

By a union bound, all the above 99/100-probability events hold with probability at least $9 / 10$. This completes the proof.

Now we can analyze the main loop of the algorithm. We either have that the empirical distribution has moments that well approximate those of $D$ or else the algorithm produces a filter that improves $S^{\prime}$. Let $\Delta\left(G, S^{\prime}\right)$ be the size of the symmetric difference between $G$ and $S^{\prime}$. Then, it suffices to show that a single iteration satisfies the following:

PROPOSITION 3.8. If we run the main loop of the algorithm above on a set $S^{\prime}$ of samples such that $\Delta\left(G, S^{\prime}\right) \leq 3 \epsilon$ for some $\epsilon$-good set $G$, then either (a) we have that $\mathbf{E}_{X \in_{u} S^{\prime}}\left[p(X)^{2}\right] \leq 1+O(\gamma+\delta+\epsilon)$, for all polynomials $p(x)$ with degree at most $d$ that have $\|p\|_{2}=1$, or else $(b)$ the loop gives a set $S^{\prime \prime} \subset S^{\prime}$ with $\Delta\left(G, S^{\prime \prime}\right) \leq \Delta\left(G, S^{\prime}\right)-\epsilon /\left(10 T_{\max }^{2}\right)$.

Proof. The case when we exit the loop is simple. For every polynomial $p(x)$ with degree at most $d$ that has $\|p\|_{2}=1$, there is a vector $v$ such that $p(x)=v^{T} \Sigma^{-1 / 2} m(x)$. Thus, we have

$$
1=\mathbf{E}_{X \sim D}\left[p(X)^{2}\right]=v^{T} \Sigma^{-1 / 2} \mathbf{E}_{X \sim D}\left[m(X) m(X)^{T}\right] \Sigma^{-1 / 2} v .
$$

Recalling that

$$
\Sigma^{-1 / 2} \mathbf{E}_{X \sim D}\left[m(X) m(X)^{T}\right] \Sigma^{-1 / 2} \geq(1+\gamma)^{-1} I,
$$

we deduce that $\|v\|_{2}^{2} \leq 1+\gamma$.
For any polynomial $p(x)=v^{T} \Sigma^{-1 / 2} m(x)$, we can write:

$$
\begin{aligned}
\mathbf{E}_{X \epsilon_{u} S^{\prime}}\left[p(X)^{2}\right]-1 & =v^{T} \Sigma^{-1 / 2} \mathbf{E}_{X \epsilon_{u} S^{\prime}}\left[m(X) m(X)^{T}\right] \Sigma^{-1 / 2} v-1 \\
& =v^{T} M v-1 \\
& \leq(1+\gamma)\left(v^{*}\right)^{T} M v^{*}-1= \\
& \leq(1+\gamma)\left(1+\lambda^{*}\right)-1 \\
& =O\left(\gamma+\lambda^{*}\right) .
\end{aligned}
$$

So, when $\lambda^{*} \leq O(\gamma+\delta+\epsilon)$, we have $\mathbf{E}_{X \in_{u} S^{\prime}}\left[p(X)^{2}\right] \leq 1+O(\gamma+\delta+\epsilon)$ for all such $p(x)$.

It remains to show that the algorithm produces a filter with the desired properties when $\lambda^{*} \geq \Omega(\gamma+\delta+\epsilon)$. Note that

$$
\left\|p^{*}\right\|_{2}^{2}=\left(v^{*}\right)^{T} \Sigma^{-1 / 2} \mathbf{E}_{X \sim D}\left[m(X) m(X)^{T}\right] \Sigma^{-1 / 2} v^{*},
$$

and so $(1+\gamma)^{-1} \leq\left\|p^{*}\right\|_{2}^{2} \leq(1-\gamma)^{-1}$. On the other hand, we have $\mathbf{E}_{X \sim{ }_{u} S^{\prime}}\left[p^{*}(x)^{2}\right]=1+O\left(\gamma+\lambda^{*}\right)$. We show that this is only possible when $\mathbf{E}_{X}\left[p^{*}(X)^{2}\right]$ is bigger under $S^{\prime}$ than under $D$, and that under these circumstances, we there exists a valid threshold for our filter.

Let $S$ be the subset of $G$ that contains the points $x$ satisfying $m(x)^{T} \Sigma^{-1} m(x) \leq T_{\max }^{2} / 2$. Then, we write $S^{\prime}=S \cup E \backslash L$ for disjoint $E$ and $L$. Thus, we have

$$
\begin{gathered}
\left|S^{\prime}\right| \cdot \mathbf{E}_{X \in_{u} S^{\prime}}\left[p^{*}(X)^{2}\right]=|S| \cdot \mathbf{E}_{X \in_{u} S}\left[p^{*}(X)^{2}\right] \\
+|E| \cdot \mathbf{E}_{X \in_{u} E}\left[p^{*}(X)^{2}\right]-|L| \cdot \mathbf{E}_{X \in_{u} L}\left[p^{*}(X)^{2}\right] .
\end{gathered}
$$

We start with the following simple lemma:

LEMMA 3.9. For all polynomials $p(x)$ with degree at most $d$ and

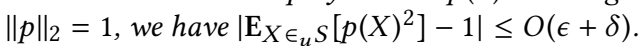

Proof. We first show that conditioning on the pruning step does not change $\mathbf{E}\left[p(X)^{2}\right]$ much. Note that, using Lemma 3.6, we have $d_{\mathrm{T} V}(D, D \|$ prune $) \leq \epsilon / 10$. We can write:

$$
\begin{aligned}
& \left|\mathbf{E}_{X \sim D}\left[p(X)^{2}\right]-\mathbf{E}_{X \sim D \mid \text { prune }}\left[p(X)^{2}\right]\right| \\
= & \left|\int_{T=0}^{\infty} T\left(\operatorname{Pr}_{X \sim D \mid \text { prune }}[|p(X)|>T]-\operatorname{Pr}_{X \sim D}[|p(X)|>T]\right) d T\right| \\
\leq & \int_{T=0}^{\infty} T \min \left\{\epsilon / 10, Q_{d}(T) /(1-\epsilon / 10)\right\} d T \\
\leq & 2 \delta .
\end{aligned}
$$

On pruned samples $x$, we have that $|p(x)| \leq T_{\max }$ by Lemma 3.5, and therefore

$$
\begin{aligned}
& \left|\mathrm{E}_{X \in \in_{u} S}\left[p(X)^{2}\right]-\mathbf{E}_{X \sim D \mid \text { prune }}\left[p(X)^{2}\right]\right| \\
= & \left|\int_{T=0}^{\infty} T\left(\operatorname{Pr}_{X \in_{u} S}[|p(X)|>T]-\operatorname{Pr}_{X \sim D \mid \text { prune }}[|p(X)|>T]\right) d T\right| \\
= & \left|\int_{T=0}^{T_{\max }} T\left(\operatorname{Pr}_{X \in_{u} S}[|p(X)|>T]-\underset{X \sim D \mid \text { prune }}{\operatorname{Pr}}[|p(X)|>T]\right) d T\right| \\
\leq & \left|\int_{T=0}^{T_{\max }} T\left(\epsilon / T_{\max }^{2}\right) d T\right| \\
= & \epsilon / 2,
\end{aligned}
$$

where we used that the set $S$ is the pruned set satisfying Condition (ii) of Definition 3.4. The triangle inequality now gives that

$\left|\mathbf{E}_{X \epsilon_{u} s}\left[p(X)^{2}\right]-1\right|=\left|\mathbf{E}_{X \epsilon_{u} s}\left[p(X)^{2}\right]-\mathbf{E}_{X \sim D}\left[p(X)^{2}\right]\right| \leq 2 \delta+\epsilon / 2$.

This completes the proof. 
We now show that the contribution of the set $L$ to the expectation of $p^{2}$ is small:

LEMMA 3.10. For all polynomials $p$ of degree at most $d$ with $\|p\|_{2}=$ 1 , we have $|L| \cdot \mathbf{E}_{X \in_{u} L}\left[p(X)^{2}\right] \leq O(\delta+\epsilon) \cdot|S|$.

Proof. Since $L \subset S$, for any event $A$, we have that $|L| \cdot \operatorname{Pr}_{L}[A] \leq$ $|S| \cdot \operatorname{Pr}_{S}[A]$, and therefore

$$
\underset{L}{\operatorname{Pr}}[A] \leq \min \{1,(|S| /|L|) \cdot \underset{S}{\operatorname{Pr}}[A]\} .
$$

Thus, we have the following sequence of inequalities:

$$
\begin{aligned}
& (|L| /|S|) \cdot \mathbf{E}_{X \in u}\left[p(X)^{2}\right] \\
= & \int_{T=0}^{T_{\max }} T(|L| /|S|) \operatorname{Pr}_{X \epsilon_{u} L}[|p(X)|>T] d T \\
\leq & \int_{T=0}^{T_{\max }} T \cdot \min \left\{|L| /|S|, \operatorname{Pr}_{X \in_{u} S}[|p(X)|>T]\right\} d T \\
\leq & \int_{T=0}^{T_{\max }} T \cdot \min \left\{3 \epsilon, \underset{X \sim D \mid \operatorname{prune}}{\operatorname{Pr}}[|p(X)|>T]+\epsilon / T_{\max }^{2}\right\} d T \\
\leq & \int_{T=0}^{T_{\max }} T \cdot \min \left\{3 \epsilon,(1+\epsilon) \cdot \operatorname{Pr}_{X \sim D}[|p(X)|>T]+\epsilon / T_{\max }^{2}\right\} d T \\
\leq & \int_{T=0}^{T_{\max }} T \cdot \min \left\{3 \epsilon,(1+\epsilon) Q_{d}(T)\right\} d T+\int_{T=0}^{T_{\max }} T\left(\epsilon / T_{\max }^{2}\right) d T \\
\leq & 3 \delta+\epsilon / 2 .
\end{aligned}
$$

This completes the proof.

As an immediate corollary, we obtain:

Corollary 3.11. For all polynomials $p$ of degree at most $d$ with $\|p\|_{2}=1$, we have that $\mathbf{E}_{X \in_{u} S^{\prime}}\left[p(X)^{2}\right] \geq 1-O(\epsilon+\delta)$.

Proof. This follows from the equation for $\mathbf{E}_{X \in_{u} S^{\prime}}\left[p(X)^{2}\right]$ similar to (1), using Lemmas 3.10 and 3.9, and the fact that

$$
|E| \cdot \mathbf{E}_{X \in_{u} E}\left[p(X)^{2}\right]>0 \text {. }
$$

Our goal is to show that our algorithm will indeed find a filter in this case, i.e, there exists $T>0$ such that $\operatorname{Pr}_{X \in \in_{u} S^{\prime}}\left[\left|p^{*}(X)\right| \geq T\right] \geq$ $4 Q_{d}(T)+3 \epsilon / T_{\max }^{2}$. We will show this by contradiction using the following intermediate lemma:

LEMmA 3.12. If for all $T>0$, we have that $\operatorname{Pr}_{X \in u} S^{\prime}\left[\left|p^{*}(X)\right| \geq T\right] \leq$ $4 Q_{d}(T)+3 \epsilon / T_{\max }^{2}$, then we have $|E| \cdot \mathrm{E}_{X \in \in_{u} E}\left[p^{*}(X)^{2}\right] \leq O(\gamma+\delta+$ $\epsilon) \cdot\left|S^{\prime}\right|$.

Proof. Since $E \subset S^{\prime}$, it follows that

$$
\begin{aligned}
\left(|E| /\left|S^{\prime}\right|\right) \cdot \operatorname{Pr}_{X \in_{u} E}\left[\left|p^{*}(X)\right|>T\right] & \leq \min \left\{|E| /\left|S^{\prime}\right|, \operatorname{Pr}_{X \in_{u} S^{\prime}}\left[\left|p^{*}(X)\right|>T\right]\right\} \\
& \leq \min \left\{3 \epsilon, 4 Q_{d}(T)+3 \epsilon / T_{\max }^{2}\right\} .
\end{aligned}
$$

Since $\left\|p^{*}\right\|_{2}^{2} \leq 1+O(\gamma)$, by a similar proof to that in Lemma 3.10 above, we have that

$$
|E| \cdot \mathbf{E}_{X \in_{u} E}\left[p^{*}(X)^{2}\right] \leq O(\gamma+\delta+\epsilon)\left|S^{\prime}\right| .
$$

Now we are ready to show that we do find a filter:
LEMmA 3.13. If $\lambda^{*} \geq \Omega(\gamma+\delta+\epsilon)$, then there exists a $T>0$ with $\operatorname{Pr}_{X \epsilon_{u} S^{\prime}}\left[\left|p^{*}(X)\right| \geq T\right] \geq 4 Q_{d}(T)+3 \epsilon / T_{\max }^{2}$.

Proof. We show the contrapositive. Suppose that there is no such $T$, then by Lemma 3.12 we get that

$$
|E| \cdot \mathbf{E}_{X \epsilon_{u} E}\left[p^{*}(X)^{2}\right] \leq O(\gamma+\delta+\epsilon)\left|S^{\prime}\right| .
$$

Now recall that $\left\|p^{*}\right\|_{2}^{2} \leq 1+O(\gamma)$. We can apply Lemma 3.9 to $p^{*}(x) /\left\|p^{*}\right\|_{2}$ to obtain

$$
|S| \cdot \mathbf{E}_{X \in_{u} S}\left[p^{*}(X)^{2}\right] \leq(1+O(\gamma))(1+O(\delta+\epsilon))|S| .
$$

Using Equation (1) and the fact that $|L| \cdot \mathbf{E}_{X \in_{u} L}\left[p^{*}(X)^{2}\right] \geq 0$, we have

$$
\begin{aligned}
& \left|S^{\prime}\right| \cdot \mathbf{E}_{X \in_{u} S^{\prime}}\left[p^{*}(X)^{2}\right] \\
\leq & (1+O(\gamma))(1+O(\delta+\epsilon))|S|+O(\gamma+\delta+\epsilon)\left|S^{\prime}\right| \\
\leq & \left|S^{\prime}\right|(1+O(\gamma+\delta+\epsilon)) .
\end{aligned}
$$

However, this implies that $\lambda^{*}=\mathbf{E}_{X \epsilon_{u} S^{\prime}}\left[p^{*}(X)^{2}\right]-1=O(\gamma+\delta+\epsilon)$, yielding the desired contradiction.

The algorithm thus finds a filter in this case. We next show that it rejects more points from $E$ than $S$, thus reducing $\Delta\left(S, S^{\prime}\right)$ :

Lemma 3.14. We have that $\Delta\left(S^{\prime \prime}, S\right) \leq \Delta\left(S^{\prime}, S\right)-\epsilon /\left(10 T_{\max }^{2}\right)$.

Proof. Using the tail bound and the goodness of $S$, we obtain that

$$
\operatorname{Pr}_{X \in_{u} S}\left[\left|p^{*}(x)\right| \geq T\right] \leq(1+\epsilon) Q_{d}(T)+3 \epsilon / T_{\max }^{2} .
$$

On the other hand, the filter rejects samples $x$ with $\left|p^{*}(x)\right| \geq T$ of which there are at least $\left(4 Q_{d}(T)+3 \epsilon / T_{\max }^{2}\right)\left|S^{\prime}\right|$ many in $S^{\prime}$. With appropriate choice of constant, we obtain that at least $2 / 3$ of the rejected samples are from $E$ and not $S^{\prime}$. A similar analysis to Claim 8.12 of [13] gives the lemma.

Since neither $S^{\prime \prime}$ nor $S^{\prime}$ contain any points $x$ with $m(x)^{T} \Sigma^{-1} m(x) \geq$ $T_{\max }^{2} / 2$, we also have $\Delta\left(S^{\prime \prime}, G\right) \leq \Delta\left(S^{\prime}, G\right)-\epsilon /\left(10 T_{\max }^{2}\right)$. This completes the proof of Proposition 3.8.

Now we analyze the case that we exit the loop. Our aim is to show the following lemma:

LEMma 3.15. For any polynomial p of degree at mostd with $\|p\|_{2} \leq$ 1 , we have that

$$
\left|\mathbf{E}_{X \in_{u} S^{\prime}}[f(X) p(X)]-\mathbf{E}_{X \sim D}[f(X) p(X)]\right| \leq O(\sqrt{\epsilon(\gamma+\delta+\epsilon)}) .
$$

Since the expectations the algorithm outputs are those over $S^{\prime}$, Lemma 3.15 implies that the linear combinations that give an approximation to $\mathbf{E}_{X \sim D}[f(X) p(X)]$ have this error, and so the algorithm is correct.

To prove Lemma 3.15, we will need to show a number of intermediate statements. Firstly, we note that the pruning step does not affect this expectation under $D$ much:

Lemma 3.16. For all polynomials $p$ of degree at most $d$ and $\|p\|_{2}=$ 1, we have: $\left|\mathbf{E}_{X \sim D}[f(X) p(X)]-\mathbf{E}_{X \sim D \mid \text { prune }}[f(X) p(X)]\right| \leq O(\epsilon+$ $\sqrt{\epsilon \delta})$. 
Proof. We have that

$$
\begin{aligned}
\mathbf{E}_{X \sim D}[f(X) p(X)]= & (1-\underset{D}{\operatorname{Pr}}[\text { prune }]) \mathbf{E}_{X \sim D \mid \text { prune }}[f(X) p(X)] \\
& +\underset{D}{\operatorname{Pr}}[\text { prune }] \mathbf{E}_{X \sim D \mid \neg \text { prune }}[f(X) p(X)] .
\end{aligned}
$$

Thus, we can write:

$$
\begin{aligned}
& \left|\mathbf{E}_{X \sim D}[f(X) p(X)]-\mathbf{E}_{X \sim D \mid p r u n e}[f(X) p(X)]\right| \\
& =\mid \underset{D}{\operatorname{Pr}}[\text { prune }] \mathbf{E}_{X \sim D \mid \text { prune }}[f(X) p(X)] \\
& +\underset{D}{\operatorname{Pr}}[\text { prune }] \mathbf{E}_{X \sim D \mid} \text { prune }[f(X) p(X)] \mid \\
& \leq \underset{D}{\operatorname{Pr}}[\text { prune }] \sqrt{\mathbf{E}_{X \sim D \mid \text { prune }}\left[p(X)^{2}\right]}+\underset{D}{\operatorname{Pr}[\text { prune }]} \sqrt{\mathbf{E}_{X \sim D \mid \neg \text { prune }}\left[p(X)^{2}\right]} \\
& \leq \underset{D}{\operatorname{Pr}}[\text { prune }] /(1-\underset{D}{\operatorname{Pr}}[\text { prune }])+\underset{D}{\operatorname{Pr}[\text { prune }]} \sqrt{\mathrm{E}_{X \sim D \mid \neg \text { prune }}\left[p(X)^{2}\right]} \\
& \leq \epsilon+\underset{D}{\operatorname{Pr}[\text { prune }]} \sqrt{\mathbf{E}_{X \sim D \mid \neg \text { prune }}\left[p(X)^{2}\right]} .
\end{aligned}
$$

We need a bound on this last term, which we obtain as follows:

$$
\begin{aligned}
& \underset{D}{\operatorname{Pr}}[\text { prune }] \mathrm{E}_{X \sim D \mid \neg \text { prune }}\left[p(X)^{2}\right] \\
& =\mathbf{E}_{X \sim D}\left[p(X)^{2}\right]-(1-\underset{D}{\operatorname{Pr}}[\text { prune }]) \mathbf{E}_{X \sim D \mid \text { prune }}\left[p(X)^{2}\right] \\
& =\int_{T=0}^{\infty} T \cdot(\underset{X \sim D}{\operatorname{Pr}}[|p(X)|>T]- \\
& (1-\underset{D}{\operatorname{Pr}[\text { prune }])} \underset{X \sim D \mid \text { prune }}{\operatorname{Pr}}[|p(X)|>T]) d T \\
& \leq \int_{T=0}^{\infty} T \cdot \min \left\{O \underset{D}{O(\operatorname{Pr}[\text { prune }])}, O\left(Q_{d}(T)\right)\right\} d T \\
& \leq O(\delta) \text {. }
\end{aligned}
$$

This gives that $\operatorname{Pr}_{D}[$ prune $] \sqrt{\mathbf{E}_{X \sim D \mid \neg \text { prune }}\left[p(X)^{2}\right]}=O(\sqrt{\epsilon \delta})$, as required.

Finally, we can bound from above the contribution of the set $E$ to the expectation of $p^{2}$ when the algorithm terminates

LEMmA 3.17. If $S^{\prime}=S \cup E \backslash L$ is the final set of samples when the algorithm terminates, then for all polynomials $p$ of degree at most $d$

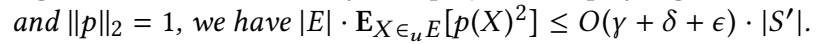

Proof. Proposition 3.8 gives that $\left|\mathbf{E}_{X \in_{u} S^{\prime}}\left[p(X)^{2}\right]-1\right| \leq O(\gamma+$ $\delta+\epsilon)$, Lemma 3.10 gives that $|L| \cdot \mathbf{E}_{X \in_{u} L}\left[p(X)^{2}\right] \leq O(\delta+\epsilon) \cdot|S|$, and Lemma 3.9 gives $\mathbf{E}_{X \epsilon_{u} S}\left[p(X)^{2}\right] \geq 1-O(\epsilon+\delta)$. Thus, we have

$|E| \cdot \mathbf{E}_{X \in_{u} E}\left[p(X)^{2}\right]$

$=\left|S^{\prime}\right| \cdot \mathbf{E}_{X \in_{u} S^{\prime}}\left[p(X)^{2}\right]+|L| \cdot \mathbf{E}_{X \in_{u} L}\left[p(X)^{2}\right]-|S| \cdot \mathbf{E}_{X \in_{u} S}\left[p(X)^{2}\right]$

$\leq\left|S^{\prime}\right| \cdot(1+O(\gamma+\delta+\epsilon))+|S| \cdot O(\delta+\epsilon)-|S| \cdot(1-O(\delta+\epsilon))$

$\leq|| S^{\prime}|-| S||+\left(|S|+\left|S^{\prime}\right|\right) \cdot O(\gamma+\delta+\epsilon)$

$\leq O(\gamma+\delta+\epsilon) \cdot\left|S^{\prime}\right|$,

recalling that $\Delta\left(S^{\prime}, S\right) \leq 2 \epsilon$.

We are now ready to prove Lemma 3.15.
Proof of Lemma 3.15: We have the following sequence of inequalities:

$$
\begin{aligned}
& \left|S^{\prime}\right| \cdot\left|\mathbf{E}_{X \epsilon_{u} S}[p(X) f(X)]-\mathbf{E}_{X \epsilon_{u} S^{\prime}}[p(X) f(X)]\right| \\
& =\left|\left(\left|S^{\prime}\right|-|S|\right) \cdot \mathbf{E}_{X \epsilon_{u} S}[p(X) f(X)]+\right| L \mid \cdot \mathbf{E}_{X \in_{u} L}[p(X) f(X)] \\
& -|E| \cdot \mathbf{E}_{X \epsilon_{u} E[p(X) f(X)] \mid} \\
& \leq|| S^{\prime}|-| S|| \cdot\left|\mathbf{E}_{X \in_{u} S}[p(X) f(X)]\right|+|L| \cdot\left|\mathbf{E}_{X \in_{u} L}[p(X) f(X)]\right| \\
& +|E| \cdot\left|\mathbf{E}_{X \in \in_{u} E}[p(X) f(X)]\right| \\
& \leq|| S^{\prime}|-| S|| \sqrt{\mathbf{E}_{X \in_{u} S}\left[p(X)^{2}\right]}+|L| \cdot \sqrt{\mathbf{E}_{X \in_{u} L}\left[p(X)^{2}\right]} \\
& +|E| \cdot \sqrt{\mathbf{E}_{X \in \epsilon_{u} E}\left[p(X)^{2}\right]} \\
& \leq O\left(\epsilon\left|S^{\prime}\right|\right) \cdot \sqrt{1+O(\delta+\epsilon)}+O(|S| \sqrt{\epsilon \cdot(\delta+\epsilon)}) \\
& +O\left(\left|S^{\prime}\right| \sqrt{\epsilon \cdot(\gamma+\delta+\epsilon)}\right) \\
& \leq O(\sqrt{\epsilon(\gamma+\delta+\epsilon)}) \cdot\left|S^{\prime}\right|,
\end{aligned}
$$

where the penultimate line uses Lemmas 3.9, 3.10, and 3.17.

\section{ACKNOWLEDGMENTS}

I. D. is supported by NSF Award CCF-1652862 (CAREER) and a Sloan Research Fellowship. D. M. K. is supported by NSF Award CCF-1553288 (CAREER) and a Sloan Research Fellowship.

\section{REFERENCES}

[1] P. Awasthi, M. F. Balcan, and P. M. Long. 2017. The Power of Localization for Efficiently Learning Linear Separators with Noise. F. ACM 63, 6 (2017), 50:1-50:27.

[2] E. Baum. 1991. A polynomial time algorithm that learns two hidden unit nets. Neural Computation 2 (1991), 510-522.

[3] J. Bruck. 1990. Harmonic analysis of polynomial threshold functions. SIAM fournal on Discrete Mathematics 3, 2 (1990), 168-177.

[4] N. Bshouty, N. Eiron, and E. Kushilevitz. 2002. PAC Learning with Nasty Noise. Theoretical Computer Science 288, 2 (2002), 255-275.

[5] C.K. Chow. 1961. On the characterization of threshold functions. In Proc. 2nd FOCS. 34-38.

[6] A. Daniely. 2015. A PTAS for Agnostically Learning Halfspaces. In Proceedings of The 28th Conference on Learning Theory, COLT 2015. 484-502.

[7] A. Daniely. 2016. Complexity theoretic limitations on learning halfspaces. In Proceedings of the 48th Annual Symposium on Theory of Computing, STOC 2016. 105-117.

[8] A. Daniely, N. Linial, and S. S.-Shwartz. 2014. From average case complexity to improper learning complexity. In Symposium on Theory of Computing, STOC 2014. 441-448.

[9] A. De, I. Diakonikolas, V. Feldman, and R. A. Servedio. 2014. Nearly Optimal Solutions for the Chow Parameters Problem and Low-Weight Approximation of Halfspaces. 7. ACM 61, 2 (2014), 11:1-11:36.

[10] M. Dertouzos. 1965. Threshold Logic: A Synthesis Approach. MIT Press, Cambridge, MA.

[11] L. Devroye and G. Lugosi. 2001. Combinatorial methods in density estimation. Springer Series in Statistics, Springer.

[12] I. Diakonikolas, P. Harsha, A. Klivans, R. Meka, P. Raghavendra, R. A. Servedio, and L. Y. Tan. 2010. Bounding the average sensitivity and noise sensitivity of polynomial threshold functions. In STOC. 533-542.

[13] I. Diakonikolas, G. Kamath, D. M. Kane, J. Li, A. Moitra, and A. Stewart. 2016. Robust Estimators in High Dimensions without the Computational Intractability. In Proceedings of FOCS'16. 655-664.

[14] I. Diakonikolas, G. Kamath, D. M. Kane, J. Li, A. Moitra, and A. Stewart. 2017. Being Robust (in High Dimensions) Can Be Practical. CoRR abs/1703.00893 (2017). https://arxiv.org/abs/1703.00893 Proceedings of ICML'17.

[15] I. Diakonikolas, G. Kamath, D. M. Kane, J. Li, A. Moitra, and A. Stewart. 2017. Robustly Learning a Gaussian: Getting Optimal Error, Efficiently. CoRR abs/1704.03866 (2017). https://arxiv.org/abs/1704.03866 Proceedings of SODA'18.

[16] I. Diakonikolas, D. M. Kane, and A. Stewart. 2016. Robust Learning of FixedStructure Bayesian Networks. CoRR abs/1606.07384 (2016).

[17] I. Diakonikolas, D. M. Kane, and A. Stewart. 2016. Statistical Query Lower Bounds for Robust Estimation of High-dimensional Gaussians and Gaussian Mixtures. 
CoRR abs/1611.03473 (2016). http://arxiv.org/abs/1611.03473 In Proceedings of FOCS'17.

[18] I. Diakonikolas, D. M. Kane, and A. Stewart. 2017. Learning Geometric Concepts with Nasty Noise. CoRR abs/1707.01242 (2017). http://arxiv.org/abs/1707.01242

[19] I. Diakonikolas, P. Raghavendra, R. A. Servedio, and L. Y. Tan. 2014. Average Sensitivity and Noise Sensitivity of Polynomial Threshold Functions. SIAM f. Comput. 43, 1 (2014), 231-253.

[20] P. Harsha, A. R. Klivans, and R. Meka. 2014. Bounding the Sensitivity of Polynomial Threshold Functions. Theory of Computing 10 (2014), 1-26.

[21] D. Haussler. 1992. Decision theoretic generalizations of the PAC model for neural net and other learning applications. Information and Computation 100 (1992), $78-150$.

[22] A. Kalai, A. Klivans, Y. Mansour, and R. Servedio. 2008. Agnostically Learning Halfspaces. SIAM 7. Comput. 37, 6 (2008), 1777-1805.

[23] D. M. Kane. 2011. The Gaussian Surface Area and Noise Sensitivity of Degree- $d$ Polynomial Threshold Functions. Computational Complexity 20, 2 (2011), 389412.

[24] D. M. Kane. 2014. The average sensitivity of an intersection of half spaces. In Symposium on Theory of Computing, STOC 2014. 437-440.

[25] D. M. Kane. 2014. The correct exponent for the Gotsman-Linial Conjecture. Computational Complexity 23, 2 (2014), 151-175.

[26] M. Kearns and M. Li. 1993. Learning in the presence of malicious errors. SIAM 7 Comput. 22, 4 (1993), 807-837.

[27] M. Kearns, R. Schapire, and L. Sellie. 1994. Toward Efficient Agnostic Learning. Machine Learning 17, 2/3 (1994), 115-141.

[28] A. Klivans, P. Long, and R. Servedio. 2009. Learning Halfspaces with Malicious Noise. Journal of Machine Learning Research 10 (2009), 2715-2740.

[29] A. Klivans, R. O'Donnell, and R. Servedio. 2008. Learning Geometric Concepts via Gaussian Surface Area. In Proc. 49th IEEE Symposium on Foundations of Computer
Science (FOCS) 541-550.

[30] A. R. Klivans, P. M. Long, and A. K. Tang. 2009. Baum's Algorithm Learns Intersections of Halfspaces with Respect to Log-Concave Distributions. In 13th International Workshop, RANDOM 2009. 588-600.

[31] E. Kushilevitz and Y. Mansour. 1993. Learning Decision Trees Using the Fourier Spectrum. SIAM 7. Comput. 22, 6 (Dec. 1993), 1331-1348.

[32] N. Linial, Y. Mansour, and N. Nisan. 1993. Constant depth circuits, Fourier transform and learnability. 7. ACM 40, 3 (1993), 607-620.

[33] W. Maass and G. Turan. 1994. How fast can a threshold gate learn?. In Computational Learning Theory and Natural Learning Systems, S. Hanson, G. Drastal, and R. Rivest (Eds.). MIT Press, 381-414.

[34] M. Minsky and S. Papert. 1968. Perceptrons: an introduction to computational geometry. MIT Press, Cambridge, MA.

[35] S. Muroga. 1971. Threshold logic and its applications. Wiley-Interscience, New York.

[36] L. Trevisan, M. Tulsiani, and S. Vadhan. 2008. Regularity, Boosting and Efficiently Simulating every High Entropy Distribution. Technical Report 103. Electronic Colloquium in Computational Complexity. Conference version in Proceedings of CCC '09.

[37] L. Valiant. 1985. Learning disjunctions of conjunctions. In Proceedings of the Ninth International foint Conference on Artificial Intelligence. 560-566.

[38] L. G. Valiant. 1984. A theory of the learnable. In Proc. 16th Annual ACM Symposium on Theory of Computing (STOC). ACM Press, 436-445.

[39] S. Vempala. 2010. Learning Convex Concepts from Gaussian Distributions with PCA. In 51th Annual IEEE Symposium on Foundations of Computer Science, FOCS. 124-130.

[40] S. Vempala. 2010. A random-sampling-based algorithm for learning intersections of halfspaces. 7. ACM 57, 6 (2010), 32:1-32:14 